\title{
Correction to: A20 prevents obesity-induced development of cardiac dysfunction
}

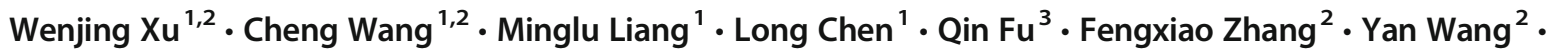 \\ Dan Huang ${ }^{1,2} \cdot$ Kai Huang ${ }^{1,2}$ (D)
}

Published online: 18 November 2020

(C) Springer-Verlag GmbH Germany, part of Springer Nature 2020

\section{Correction to: J Mol Med (2018) 96:159-172}

https://doi.org/10.1007/s00109-017-1608-3

There was an error in Fig. 6a during the proofing stage which has since been corrected. The corrected figure is presented in this paper.

The online version of the original article can be found at https:/doi.org/ 10.1007/s00109-017-1608-3

Dan Huang

joy_huangdan@yahoo.com.cn

$\triangle$ Kai Huang

huangkaiunion@gmail.com

1 Clinic Center of Human Gene Research, Union Hospital, Tongji Medical College, Huazhong University of Science and Technology, 1277 Jiefang Ave, Wuhan 430000, China

2 Department of Cardiology, Union Hospital, Tongji Medical College, Huazhong University of Science and Technology, Wuhan, China

3 Department of Pharmacology, Tongji Medical College, Huazhong University of Science and Technology, Wuhan, China 
a

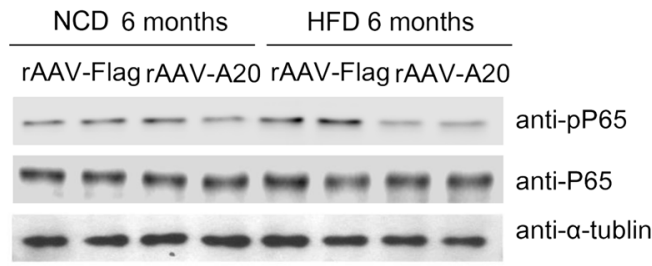

C
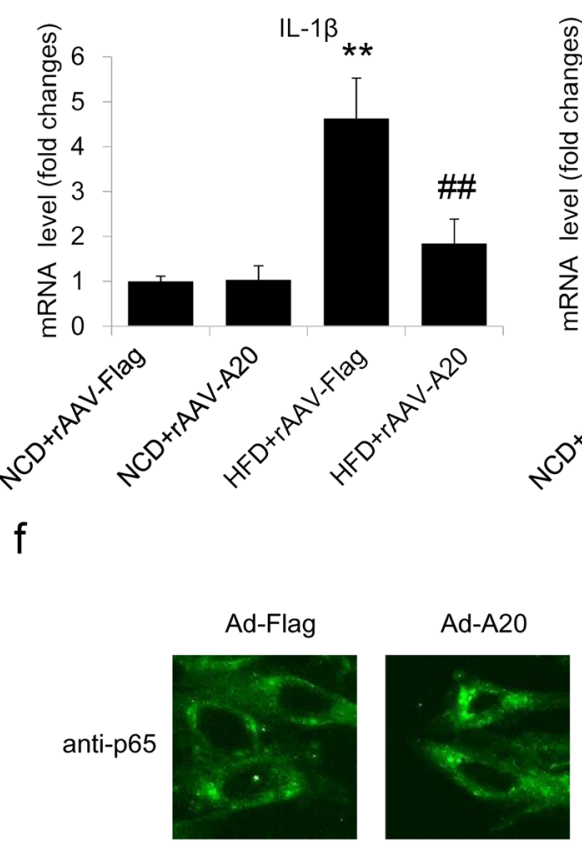

$\mathrm{h}$
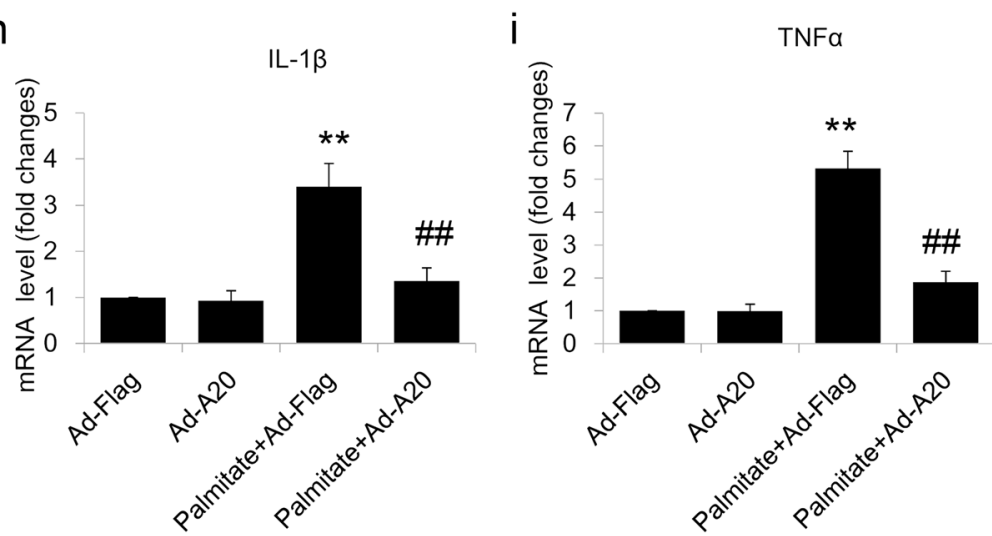
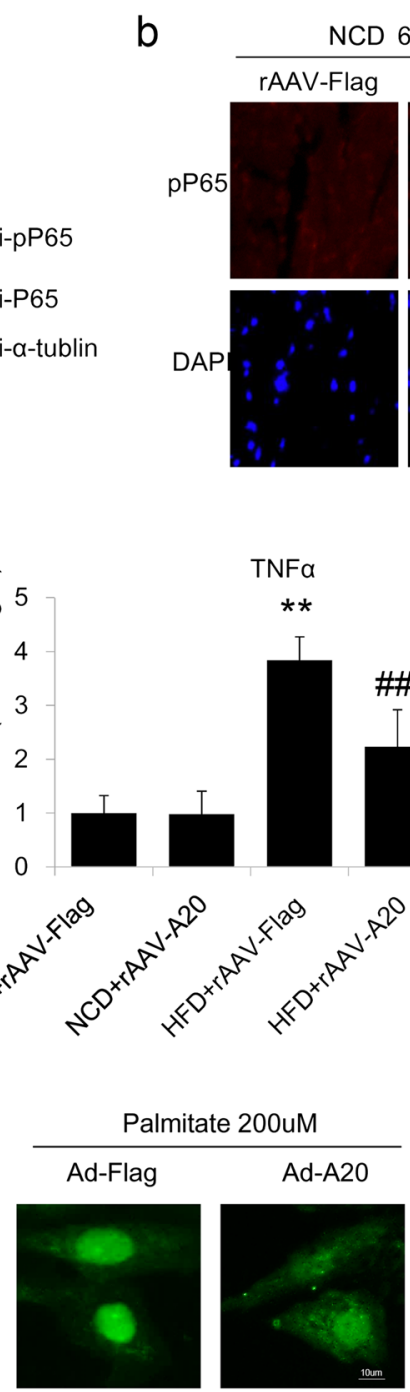

d

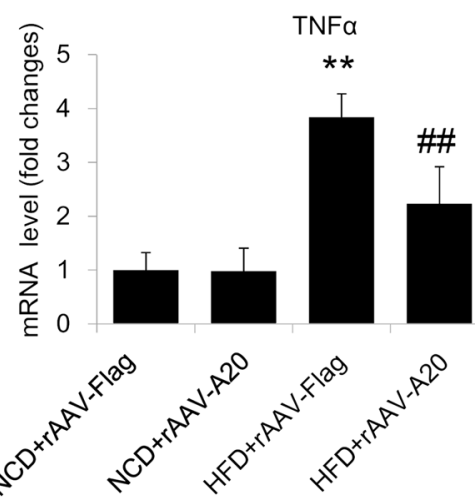

Palmitate 200uM
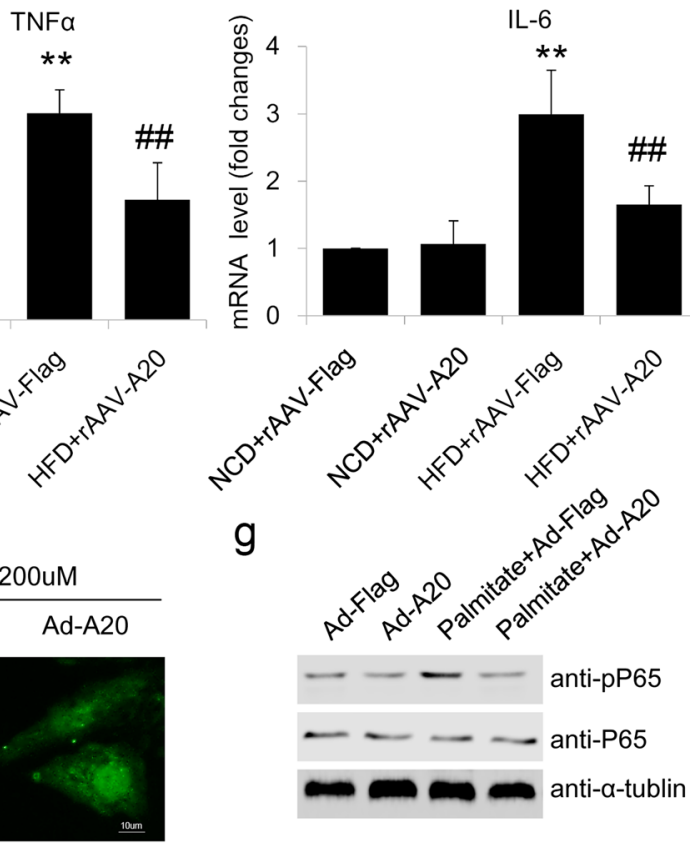

e

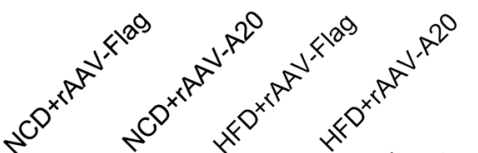

g

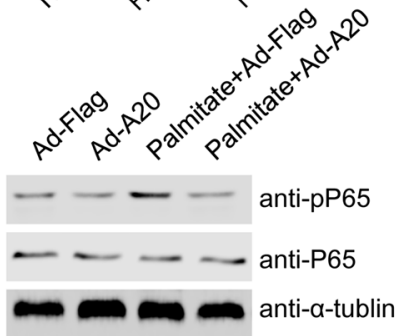

j IL-6

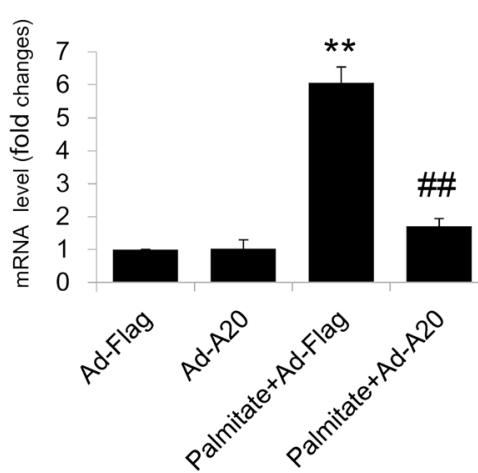

Fig. 6 A20 suppressed inflammatory cytokines production and NF- $\mathrm{BB}$ activation a Cardiac proteins were extracted from heart tissues from each group and were subjected to western blot analysis for pP65 and total P65 determination. b Immunofluorescence staining of p65 in heart tissues. Real-time PCR for (c) IL-1 $\beta$, d TNF- $\alpha$, and e IL-6 in heart tissues from each group. $N=8$ mice for each group. ${ }^{* *} P<0.01$ vs. NCD+ rAAV9Flag; ${ }^{\# \#} P<0.01$ vs. HFD+ rAAV9-Flag. NRCMs were infected with Ad-
A20 and then incubated with $200 \mu \mathrm{M}$ palmitate for $48 \mathrm{~h}$. f Immunofluorescence was performed to detected p65 translocation and pictures were taken under a fluorescence microscope. $\mathbf{g}$ The cell lysates were collected, and pP65 and total P65 proteins were detected by western blot analysis using $\alpha$ - tubulin as a loading control. The mRNA levels of $\mathbf{h}$ IL- $1 \beta$, i TNF- $\alpha$, and j IL- 6 were examined by real-time PCR. ${ }^{* *} P<0.01$ vs. Ad- Flag; ${ }^{\# \#} P<0.01$ vs. palmitate + Ad-Flag 